\title{
Deletion of chromosome $4 q$ predicts outcome in Stage II colon cancer patients
}

\author{
R. P. M. Brosens • E. J. T. H. Belt • J. C. Haan • \\ T. E. Buffart • B. Carvalho • H. Grabsch • P. Quirke • \\ M. A. Cuesta $\cdot$ A. F. Engel $\cdot$ B. Ylstra $\cdot$ G. A. Meijer
}

Accepted: 1 May 2010 / Published online: 30 June 2011

(C) The Author(s) 2011. This article is published with open access at Springerlink.com

\begin{abstract}
Background Around $30 \%$ of all stage II colon cancer patients will relapse and die of their disease. At present no objective parameters to identify high-risk stage II colon cancer patients, who will benefit from adjuvant chemotherapy, have been established. With traditional histopathological features definition of high-risk stage II colon cancer patients is inaccurate. Therefore more objective and robust markers for prediction of relapse are needed. DNA copy number aberrations have proven to be robust prognostic markers, but have not yet been investigated for this specific
\end{abstract}

Array data availability: GEO accession number GSE17181.

This paper is a reprint from 'Deletion of chromosome $4 \mathrm{q}$ predicts outcome in Stage II colon cancer patients, R.P.M. Brosens, E.J.T.H. Belt, J.C. Haan, T.E. Buffart, B Carvalho, H. Grabsch, P Quirke, M.A. Cuesta, A.F. Engel, B. Ylstra and G.A. Meijer' originally published in Analytical Cellular Pathology/Cellular Oncology, Volume 33, number 2, 2010, pp. 95 - 104, IOS Press.

R. P. M. Brosens • E. J. T. H. Belt • M. A. Cuesta Department of Surgery, VU University Medical Centre,

Amsterdam, the Netherlands

J. C. Haan · T. E. Buffart • B. Carvalho $\cdot$ B. Ylstra •

G. A. Meijer $(\triangle)$

Department of Pathology, VU University Medical Centre,

PO Box 7057, 1007 MB, Amsterdam, The Netherlands

e-mail: ga.meijer@vumc.nl

H. Grabsch • P. Quirke

Department of Pathology and Tumour Biology,

Leeds Institute of Molecular Medicine, University of Leeds,

Leeds, UK

A. F. Engel

Department of Surgery, Zaans Medical Centre,

Zaandam, the Netherlands group of patients. The aim of the present study was to identify chromosomal aberrations that can predict relapse of tumor in patients with stage II colon cancer.

Materials and methods DNA was isolated from 40 formaldehyde fixed paraffin embedded stage II colon cancer samples with extensive clinicopathological data. Samples were hybridized using Comparative Genomic Hybridization (CGH) arrays to determine DNA copy number changes and microsatellite stability was determined by PCR. To analyze differences between stage II colon cancer patients with and without relapse of tumor a Wilcoxon rank-sum test was implemented with multiple testing correction.

Results Stage II colon cancers of patients who had relapse of disease showed significantly more losses on chromosomes 4 , $5,15 \mathrm{q}, 17 \mathrm{q}$ and $18 \mathrm{q}$. In the microsatellite stable (MSS) subgroup $(n=28)$, only loss of chromosome $4 \mathrm{q} 22.1-4 \mathrm{q} 35.2$ was significantly associated with disease relapse $(P<0.05$, FDR $<0.15$ ). No differences in clinicopathological characteristics between patients with and without relapse were observed.

Conclusion In the present series of MSS stage II colon cancer patients losses on 4q22.1-4q35.2 were associated with worse outcome and these genomic alterations may aid in selecting patients for adjuvant therapy.

Keywords DNA copy number changes · Stage II · Colon cancer $\cdot$ Prognosis
Abbreviations
CGH Comparative Genomic Hybridization
FFPE Formalin fixed paraffin embedded
MSS micro satellite stable
MSI micro satellite instable
CRC Colorectal cancer 


\section{Introduction}

Worldwide, colorectal cancer (CRC) is the third most common cancer affecting more than 940,000 patients annually. Nearly 500,000 patients die from the consequences of CRC each year (www.who.int) [43]. In the Netherlands in 2006, 11231 patients were diagnosed with CRC and 4709 CRC related deaths occurred [40]. The incidence of CRC is relatively high in the western world and steadily increases each year [40]. Two thirds of all CRCs occur in the colon and one third is located in the rectum. Patients with colon tumors are treated differently and have a different prognosis compared to rectal cancer patients.

Staging is traditionally based on pathologist's evaluation of tumor extent, lymph nodes and distant metastasis, all included in the TNM classification [34] and therapeutic decisions are based on this system. Upon resection of the primary tumor, some $40 \%$ of all colon cancers appear to be stage II (TNM/UICC) [34]. Of patients with stage II colon cancer 20-30\% will relapse, i.e. develop distant metastasis, and these patients will die of their disease. Five-year survival rates for stage II colon vary between $50 \%$ and $90 \%$. Patients with stage III colon cancer will face relapse of tumor during follow-up in $60 \%$ of cases and chemotherapy can reduce recurrence rates with $40-50 \%$ [45]. Although postoperative chemotherapy is standard for stage III colon cancer it is not for stage II colon cancer [5, 14]. Nevertheless, it has been shown that subgroups of patients with stage II colon cancer can benefit from adjuvant chemotherapy [24]. In fact, high risk stage II colorectal cancer patients have a worse prognosis than single node positive stage III colorectal cancer patients [28]. Better prediction of relapse as an indication for the need of adjuvant chemotherapy is thus required for stage II colorectal cancer [5]. Histopathological and clinical variables are most frequently used for identifying high risk stage II colon cancers. Peritoneal involvement, extramural vascular invasion, tumor perforation, male gender, bowel obstruction, number of nodes harvested and presence of lymph node micro metastasis have all been identified as negative prognostic factors in stage II colon cancer [16, 22, 30]. Notwithstanding this extensive body of work, these histopathological factors have not yet provided an established basis for accurately identifying patients at high risk for relapse in a clinical setting. Molecular markers can help to select stage II colon cancer patients with worse prognosis. Gene expression profiles have been able to identify stage II colon cancer patients at high risk for relapse [4, 15]. However, RNA is an instable molecule and its expression subject to environmental and circadian rhythms. In contrast, DNA is more stable and can be obtained for copy number analysis from formalin fixed paraffin embedded (FFPE) material which is frequently the only material available in routine clinical practice. DNA copy number aberrations are a hallmark of cancer. These chromosomal aberrations are less frequent in micro satellite instable (MSI) tumors compared to micro satellite stable (MSS) tumors. The latter group is also referred to Chromosomal Instable (CIN) and accounts for $85 \%$ of all colorectal tumors. MSS CRCs have a worse outcome than MSI CRCs [41].

In CRC, chromosomal aberrations, important for tumor progression and prognosis, have been studied [12, 18-20, $23,27,29,32]$, but to which extent specific chromosomal changes have prognostic value specifically in stage II colon cancer patients has only been analyzed in limited detail. Therefore, the present study analyzed genome wide DNA copy number changes using high resolution oligonucleotide based array CGH for identifying chromosomal aberrations that may be used as prognostic markers for patients with stage II colon cancer.

\section{Materials and methods}

\subsection{Patient and sample selection}

Forty patients operated between 1990 and 2000 for stage II colon cancer (pT3 or pT4, pN0, pM0, R0 TNM classification, fifth edition [34]) were selected for this study, 16 with and 24 without relapse. Seventeen patients underwent resection of their primary tumor at the John Goligher Colorectal Unit, Leeds General Infirmary (UK) and 23 patients at the Zaans Medical Centre (NL). Medical records of all patients were reviewed retrospectively to obtain clinical data, patient characteristics and follow-up data. None of the patients received postoperative chemotherapy or radiotherapy. Tumor relapse was defined as the occurrence of distant metastasis, confirmed by ultrasound, CT scan and/or histology within 36 months. Haematoxylin \& eosin (H\&E) stained sections were reviewed by a pathologist for TNM tumor stage [34], differentiation grade, number of nodes assessed tumor perforation, extramural vascular invasion (EMVI) and peritoneal involvement. All samples were used in compliance with the respective institutional ethical regulations for surplus material and use of material from Leeds General Infirmary (UK) was approved by the Leeds (West) research ethics committee, unique identifier CA02/014.

\subsection{DNA isolation from formalin-fixed and paraffin-embedded (FFPE) tissue blocks}

DNA was isolated from FFPE colon cancer tissue of 40 samples. Corresponding normal mucosa DNA was isolated from 37 of the samples as a reference and was obtained from the resection margins or at least $1 \mathrm{~cm}$ distance from the tumor. For 3 cases no normal mucosa was available and 
a pool of reference DNA isolated from blood obtained from eighteen healthy males was used. For each tumor an area containing at least $70 \%$ of tumor cells was marked on the slides. Of the FFPE blocks 4 to $610 \mu \mathrm{m}$ sections were cut, deparaffinized and macro dissected. Isolation of DNA was performed as previously described [42], including incubation with sodium thiocyanate $(1 \mathrm{M}, \mathrm{CNNaS})$, proteinase $\mathrm{K}$ treatment and purification using a column based method (QIAamp microkit; Qiagen, Hilden, Germany).

\subsection{Microsatellite stability}

All samples were analyzed for Microsatellite instability (MSI) using MSI Analysis System, Version 1.1 according to manufacturer's instructions (Promega, Madison, USA). This PCR based assay is using 5 mononucleotide markers to determined MSI status. PCR product were separated by capillary electrophoresis using ABI 3130 DNA sequencer and output data were analyzed using the accompanying package GeneScan 3100 (Applied Biosystems, Foster City. CA, USA). Tumors were classified as MSI when instability of two or more markers was seen. When a single or no instable markers were seen, tumors were considered as micro satellite stable (MSS).

\subsection{Chromosomal copy number analysis by array} comparative genomic hybridization (array CGH)

Labeling and hybridization was done as previously described [6]. Briefly, $500 \mathrm{ng}$ of genomic DNA of tumor and reference was labeled with either Cyanine 3-UTP (Сy3) or Cyanine 5UTP (Cy5) nucleotide mixture, respectively, according to manufacturer's instructions (CGH labeling Kit for Oligo Arrays, Enzo Life Sciences, Farmingdale, NY, USA) and purified using the QIAquick PCR Purification Kit (Qiagen, Westburg, Leusden, NL). Labeled tumor and (matched) reference DNAs were mixed prior to hybridization onto Agilent 4x44K oligonucleotide arrays (Agilent Technologies, Palo Alto, USA). Each slide consists of 4 arrays, each containing 45220 in-situ synthesized 60-mer oligonucleotides representing 42494 unique biological features distributed over the genome. Immediately after hybridization the slides were scanned using microarray scanner G2505B (Agilent technologies, Palo Alto, USA) and image analysis was performed using feature extraction software (version 9.1, Agilent Technologies, Palo Alto, USA). The Agilent CGHv4_91 protocol was applied using default settings. Oligonucleotides were mapped according to the human genome build NCBI 36 (May 2006). Of both Cy3 and Cy5 channels, local background was subtracted from the median intensities. The $\log 2$ tumor to normal intensity ratio was calculated for each spot and normalized against the median of the ratios of all autosomes.
2.5 Statistical analysis of genomic profiles

Analysis of array CGH data was done in the statistical computing language $\mathrm{R}$, version 2.6.1 (http://www.r-project. org). Chromosomal copy number losses and gains were identified using the package CGHcall [37] with cellularity set to 0.7 and median normalization. To reduce the dimension of the array CGH data set without loss of information, regions were defined as previously described [39].

To calculate significance of DNA copy number differences between patients who did and did not relapse, a WilcoxonMann-Whitney two sample test was used, implemented in the software package CGHmultiarray, that provides multiple comparison correction [38]. Differences were analyzed for all patients and for MSS colon cancer patients separately. P-values of $<0.05$ and false discovery rates, i.e. the expected proportion of false positives among the DNA copy numbers claimed to be correlated with outcome, of $<0.15$ were considered to be statistical significant [13].

Statistical analysis of associations with histopathological and clinical variables was performed using SPSS software (SPPS for Windows, version 15.0, SPSS Inc.Chicago, IL, USA). Mann-Whitney U test and Chi-square test were used for analyzing differences in continuous or categorical variables, respectively. For correlations with overall survival (OS) and Disease free survival (DFS) univariate survival analysis with log rank statistics was used and Kaplan-Meier curves were constructed. To determine independent effects of clinical variables and DNA copy number changes, multivariate Cox proportional hazard analysis was performed. OS and DFS were defined as time from surgery to date of death due to all causes or to date of first evidence of distant metastasis. P-values $<0.05$ were considered as statistical significant.

\section{Results}

\subsection{Patient and tumor characteristics}

Patient and tumor characteristics of 40 stage II colon cancer patients are listed in Table 1. Twenty-eight tumors were MSS and 12 MSI. Relapse of tumor occurred in 14 MSS patients and 2 MSI patients $(P=0.05)$. No significant differences in age, gender, number of nodes assessed, differentiation grade, T-stage and other histopathological variables between patients who relapsed and who did not were seen (Table 1). Mean follow-up time for all patients was 73 months (range 2-155). A trend was seen for DFS of MSI patients being better compared to MSS patients, although this was not significant (5-year DFS $80 \%$ versus $50 \%, P=0.06$ ) (Fig. 1). Survival time for MSS patients with 
Table 1 Patients and tumor characteristics of stage II colon cancers $(N=40)$

\begin{tabular}{|c|c|c|c|c|}
\hline & & No Relapse $(n=24)$ & Relapse $(n=16)$ & P-value \\
\hline Mean Age (years) (range) & & $72(49-91)$ & $73(54-90)$ & $\mathrm{ns}^{\$}$ \\
\hline Mean number of nodes assessed (range) & & $8.5(3-17)$ & $7.7(1-22)$ & $\mathrm{ns}^{\$}$ \\
\hline \multicolumn{5}{|l|}{ Gender } \\
\hline & Female & 12 & 10 & \multirow[t]{2}{*}{$\mathrm{ns}^{\S}$} \\
\hline & Male & 12 & 6 & \\
\hline \multicolumn{5}{|l|}{ Location } \\
\hline & Right -sided* & 18 & 10 & \multirow[t]{2}{*}{$\mathrm{ns}^{\S}$} \\
\hline & Left -sided ${ }^{\#}$ & 6 & 6 & \\
\hline \multirow[t]{2}{*}{ T-stage } & $\mathrm{T} 3$ & 25 & 17 & \multirow[t]{2}{*}{$\mathrm{ns}^{\S}$} \\
\hline & $\mathrm{T} 4$ & 1 & 1 & \\
\hline \multicolumn{5}{|l|}{ Differentiation } \\
\hline & Poor & 2 & 3 & \multirow[t]{2}{*}{$\mathrm{ns}^{\S}$} \\
\hline & Moderate & 22 & 13 & \\
\hline \multicolumn{5}{|l|}{ Tumor perforation } \\
\hline & Yes & 0 & 0 & \multirow[t]{2}{*}{$\mathrm{ns}^{\S}$} \\
\hline & No & 24 & 16 & \\
\hline \multicolumn{5}{|l|}{ Peritoneal involvement } \\
\hline & Yes & 1 & 1 & \multirow[t]{2}{*}{$\mathrm{ns}^{\S}$} \\
\hline & No & 23 & 15 & \\
\hline \multicolumn{5}{|l|}{ EMVI } \\
\hline & Yes & 2 & 2 & \multirow[t]{3}{*}{$\mathrm{ns}^{\S}$} \\
\hline & No & 19 & 4 & \\
\hline & Unknown & 13 & 10 & \\
\hline \multicolumn{5}{|l|}{ MSI } \\
\hline & Yes & 10 & 2 & \multirow[t]{2}{*}{$0.05^{\S}$} \\
\hline & No & 14 & 14 & \\
\hline
\end{tabular}

*Right (caecum, ascending colon, transverse colon, hepatic flexure) $)^{\#}$ Left ( splenic flexure, descending colon, sigmoid colon)

EMVI $=$ Extramural vascular invasion

Ns = not significant

\$ Mann-Whitney U-test; ${ }^{\S}$ Chi-square

$(n=14)$ or without $(n=14)$ relapse was 23 months (range 259) versus 104 months (range 5-155), respectively.

3.2 DNA copy numbers aberrations in MSS and MSI stage II colon cancer patients

CGH profiles of 40 stage II colon cancers patients showed copy number aberrations consistent with those reported in literature $[7,8,10,35,36]$. The mean fraction of the genome altered was $15.3 \%$ (range $0-44 \%$ ), and was significantly higher for MSS tumors $(19.4 \%$, range 0 $44 \%)$ compared to MSI tumors $(5.9 \%$, range $0-18 \%)(\mathrm{P}<$ $0.001)$. The amount of losses versus gains was balanced in the MSS tumors, but MSI tumors showed more gains than losses; gains $4.8 \%(0-16.2 \%)$ versus losses $1.1 \%(0-$ $3.1 \%$ ). Gains in MSI tumors predominately occurred at 8q24.3 (4 out of 12) and 9p21.3 (4 out of 12) and losses at 6p22.1
(4 out of 12), 16p13.2 (5 out of 12) and 17p13.1 (3 out of 12). For MSS tumors the highest frequency of aberrations (in more than 12 out of 28 patients) were gains of chromosome 7p22-p11, 7q11, 7q22, whole chromosome 13 and 20 and losses of chromosome 8p23.1-p12, 15q13.3-q26.1, 17p13.2-p11.2 and 18p11.3-q22.3. DNA copy number changes of MSI and MSS tumors are summarized in Fig. 2a and b.

3.3 Differences in DNA copy numbers aberrations between stage II colon cancer patients with and without relapse

Overall, significant differences were observed between patients without or with relapse, with $11.2 \%$ versus $21.4 \%$ of the genome altered $(P=0.005)$. This was primarily reflected in the amount of losses which was $10.9 \%$ of the genome for the patients which relapsed versus $3.9 \%$ for the 
Fig. 1 Kaplan-Meier analysis of disease-free survival for 40 stage II colon cancer patients stratified by MSI / MSS status. MSI colon tumors showed a trend towards better disease-free survival compared to MSS patients $(P=0.06)$

Fig. 2 Percentage of chromosomal gains and losses measured by array CGH in (a) $28 \mathrm{MSS}$ and (b) 12 MSI stage II colon cancers patients. X-axis displays 44,000 oligo-nucleotides in genomic order (chromosomes 122). Y-axis displays percentage of tumor with gains $(>0)$ or losses $(<0)$. Boundaries of chromosomes are indicated by black vertical lines and locations of centromeres are indicated by dotted lines
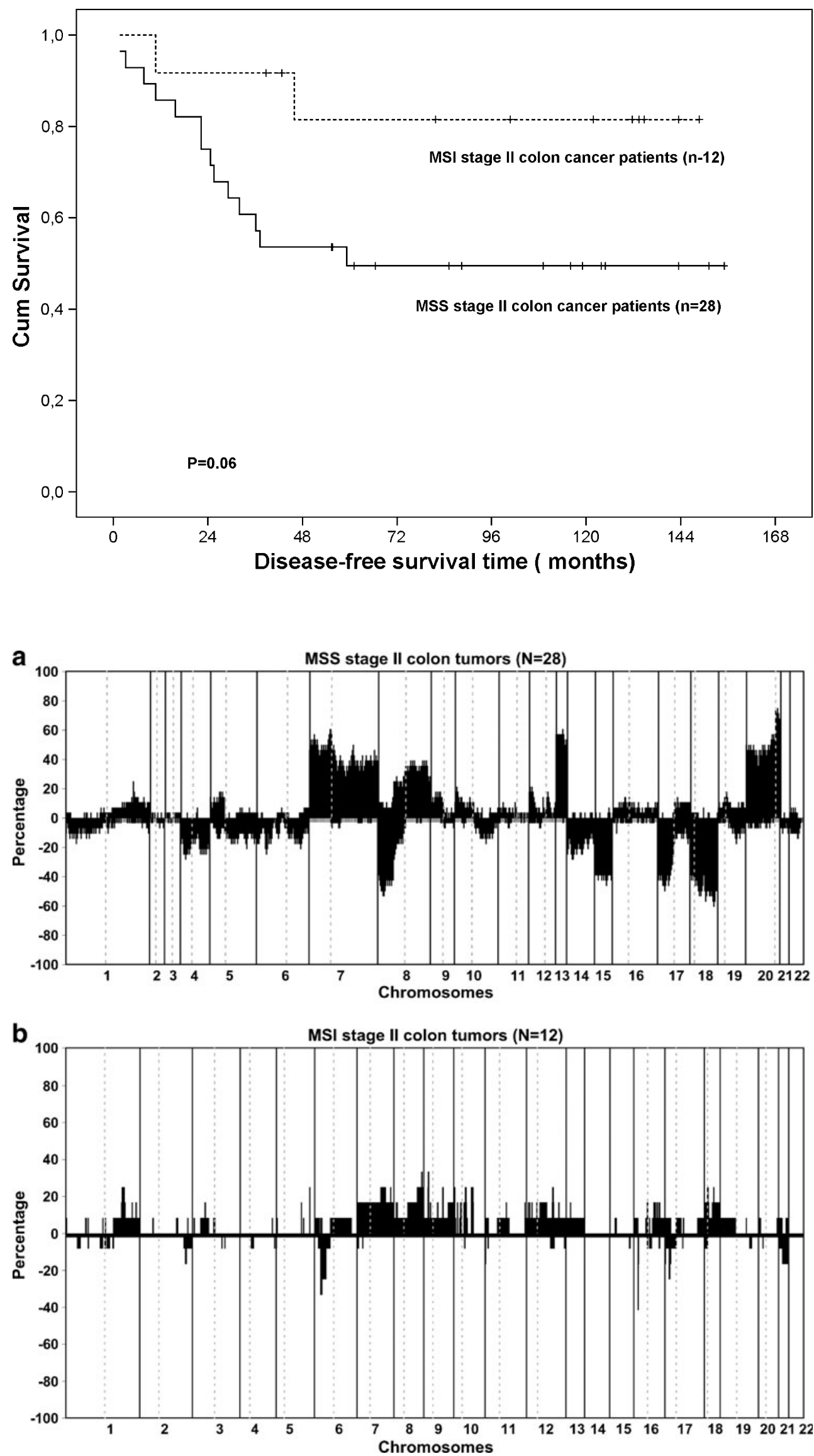
patients which remained disease free $(P=0.002)$. The chromosomal region(s) which significantly differed between patients with or without relapse were losses on chromosome 4p16.1-p12 (30 Mb), 4q12-q35.2 9 (127 Mb), 5p14 (9 Mb), 5q11.2-q14.2 (21 Mb), 5q32-q34 (0.86 Mb), 15q11.2-q26.1 $(21 \mathrm{Mb}), 17 \mathrm{q} 21.1(0.48 \mathrm{Mb}), 18 \mathrm{q} 12.1-\mathrm{q} 12.3(15 \mathrm{Mb})$ and 18q21.1-q22.13 (6 Mb). No significant difference was observed in the amount of gains, $7.3 \%$ of the genome altered for patients who did not relapse versus $10.5 \%$ for patients that $\operatorname{did}(P=0.11)$.

Also among the 28 MSS patients, more aberrations were observed in patients who relapsed $(P=0.03)$, being mainly losses. Loss of chromosome 4q22.1-4q35.2 (90.5 Mb) was the most common aberration, being more frequently deleted in patients with relapse of tumor. Loss of $4 \mathrm{q} 22.1-4 \mathrm{q} 35.2$ was seen in $43 \%$ to $50 \%(P=0.01$ to $P=0.004)$ of patients who relapsed, depending on which boundaries for the lost region were used. An overview of DNA copy number changes in MSS patient who either relapsed or not, is presented in Fig. 3. The number of MSI patients was too small to further stratify and analyze differences between either or not relapse occurred.

Both OS and DFS were significantly worse for patients with loss of chromosome 4q22.1-4q35.2 or part of that region. Considering any loss on chromosome 4q22.1$4 \mathrm{q} 35.2$ predicted worse OS and DFS survival (5-year OS $70 \%$ versus $5 \%, P<0.001 ; 5$-year DFS $75 \%$ versus $5 \%, P<$ 0.001 ) (Fig. 4). Cox proportional hazard model demonstrated that any loss on chromosome 4q22.1-4q35.2 was an independent prognostic factor for worse DFS (HR $=15.4$, $P<0.001)$. Age $>72.5$ year $(\mathrm{HR}=6.7, P=0.04)$ and any loss on chromosome 4q22.1-4q35.2 $(\mathrm{HR}=16.9, P<0.001)$ were independent prognostic factors for OS.

\section{Discussion}

A substantial part of stage II colon cancer patients currently develop distant metastasis after resection of their primary tumor and subsequently die of their disease. Nevertheless,
Fig. 3 Percentage of chromosomal gains and losses measured by array CGH in $28 \mathrm{MSS}$ stage II colon cancer patients without (a) and with (b) relapse of disease. X-axis displays 44,000 oligo-nucleotides in genomic order (chromosomes 1-22). Y-axis displays percentage of tumor with gains $(>0)$ or losses $(<0)$. Boundaries of chromosomes are indicated by black vertical lines and the location of centromeres are indicated by dotted lines
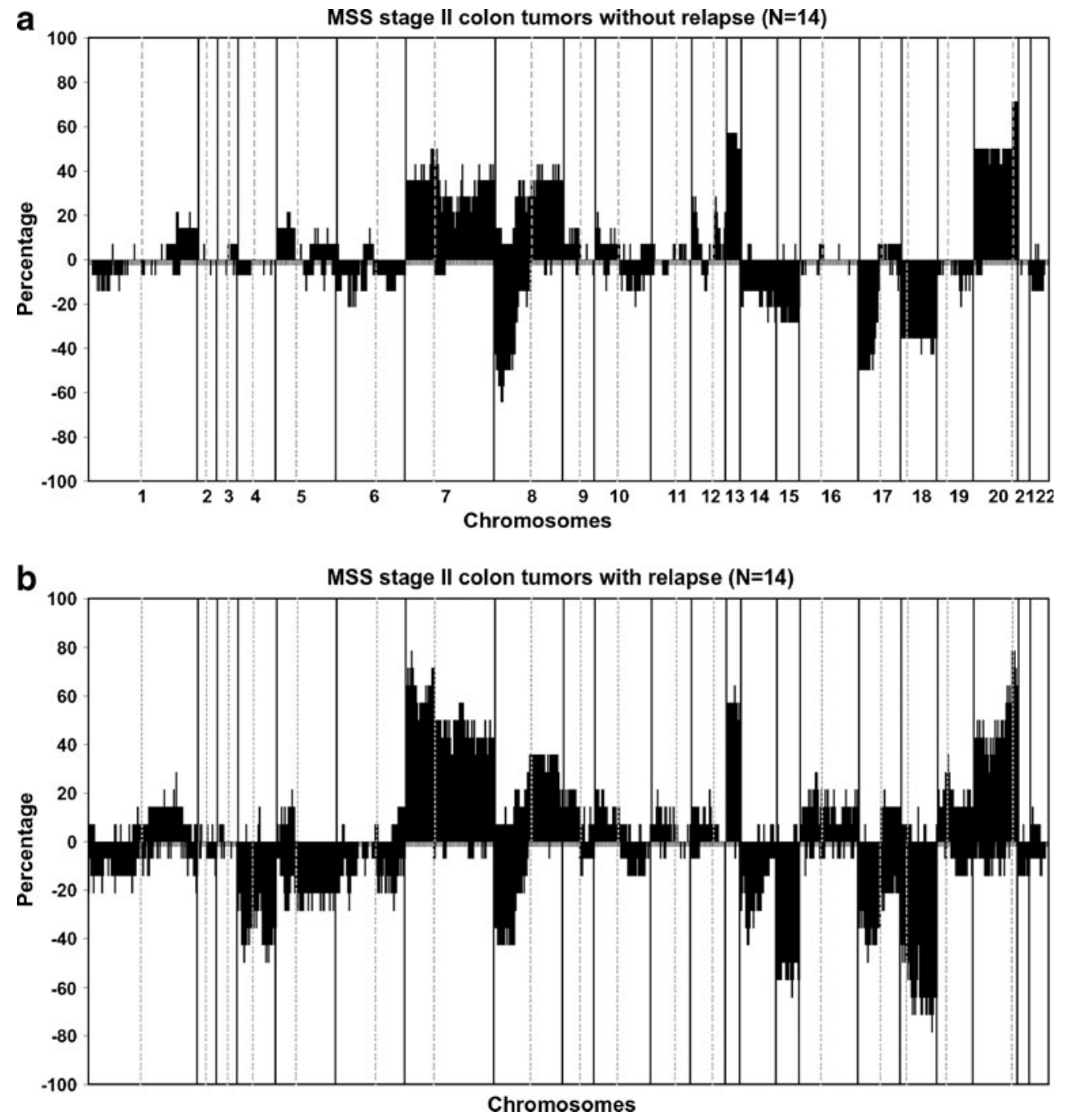
Fig. 4 Kaplan-Meyer diseasefree survival plot of $28 \mathrm{MSS}$ stage II colon cancer patients stratified for any loss of chromosome 4q22.1-q35.2. Stage II colon tumors with any loss on chromosome 4q22.1-q35.2 showed worse disease-free survival than without losses on 4q22.1-q35.2 $(P=0.004)$

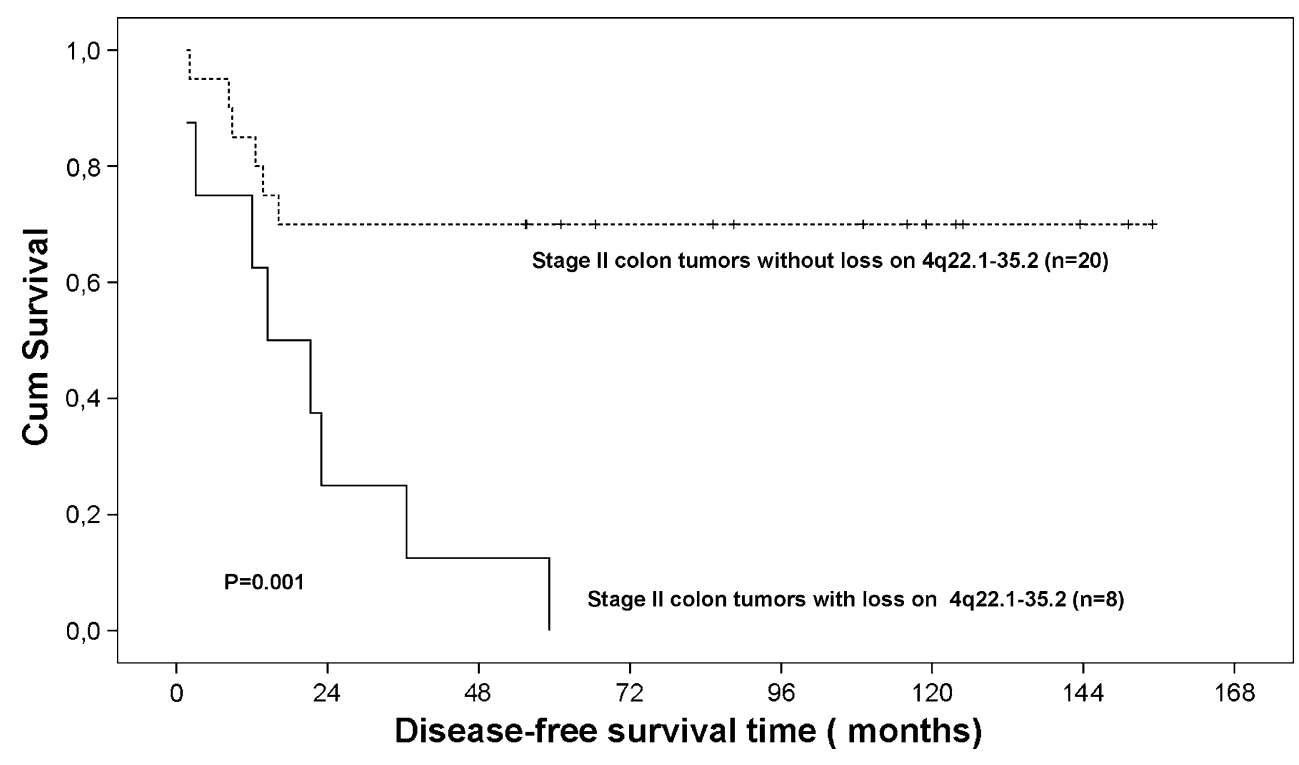

the American Society of Clinical Oncology (ASCO) [5] and the National Comprehensive Cancer Network (NCCN) [44] independently recommended against routine administration of adjuvant therapy in stage II disease, due to a lack of evidence for the benefit of survival [5, 14, 17, 24, 45]. Notwithstanding, it has been suggested that subgroups of stage II patients will benefit from adjuvant therapy. At present no objective parameters for selecting these patients are established [10, 28]. Hence, objective and robust prognostic markers are desirable to select and stratify stage II colon cancer patients at high risk for relapse. The present study found in stage II colon cancer patients deletion of chromosome 4q to be associated with relapse of disease. This conclusion is consistent with literature $[1-3,11,12$, 19] and suggests that it could be worthwhile further exploring whether patients with loss of chromosome $4 \mathrm{q}$ should be considered for adjuvant therapy.

Moreover, in the present study significantly more losses of chromosome 4p, 4q, 5p, 5q, 15q, 17q, and 18q were seen in tumors of patients who had cancer relapse. This observation is validated by the results published by Sheffer et al who linked worse prognosis to a simultaneous deletion of $4 p, 15 q$ and $18 q$ in colorectal cancer [32]. Forty percent (12 of 40) of tumors were MSI and had $5.8 \%$ of their genome altered, which were primarily gains $(4.8 \%)$. This is in accordance with results reported by Trautman et al. who reported $2.3 \%$ gain versus $0 \%$ loss in 23 MSI and $17.2 \%$ gain versus $19.9 \%$ loss in 23 MSS sporadic colon cancers analyzed by BAC array CGH [35].

When MSS patients were analyzed, only losses in the region of 4q22.1-35.2 occurred significantly more frequent in patients who relapsed compared to those who did not. Losses of $4 p, 5 p, 5 q, 15 q, 17 q$, and $18 q$ were associated but not significantly related to relapse when only MSS patients were analyzed. A likely explanation for this could be a limited sample size and hence lack of power to detect associations with e.g. 18q loss at a statistically significant level. Loss of $18 \mathrm{q}$ as prognostic marker in stage II CRC have been reported previously based on loss of heterozygosity analysis [21, 25]. As no aberrations on chromosome 4 were seen in MSI tumors, loss of $4 \mathrm{q}$ appears particularly relevant for predicting relapse in patients with stage II MSS colon tumors.

Numerous studies have analyzed the relation of DNA copy number changes and clinical outcome in colorectal cancer patients. In only a small number of studies the prognostic value of chromosomal aberrations limited to patients with early stage colon cancer have been analyzed. In 70 stage I and II MSI and MSS colorectal cancer patients, loss of chromosome $4 \mathrm{p}$ was an independent prognostic factor, and loss of chromosomes 4 and $14 \mathrm{q}$ were associated with a worse prognosis [2]. This study used $\mathrm{BAC}$ array $\mathrm{CGH}$ and reported minimally deleted regions at $4 \mathrm{q} 24-28$ and $4 \mathrm{q} 32-35$, which is consistent with the findings in the present study [2]. Additional detailed analysis of this group of patients using low resolution loss of heterozygosity $(\mathrm{LOH})$ analysis showed that in particular loss of $4 \mathrm{p} 16,4 \mathrm{q} 31.1$ and $4 \mathrm{q} 33.1$ was related to prognosis, further validating our findings [1]. When chromosomal aberrations were studied with CGH in stage I-IV colorectal tumors, loss of chromosome $4 \mathrm{q}$ resulted in shorter survival times [11], loss of 4q3234 was associated with metastasis [3] and loss of $4 \mathrm{q} 35$ and $4 q 31.3$ correlated with shorter disease-specific survival [19]. A meta-analysis of $31 \mathrm{CGH}$ studies encompassing copy number profiles of 373 colorectal tumors and 102 liver metastasis, showed loss of chromosome 4 to be related to progression from primary tumor to distant metastasis [12]. Loss of chromosome $4 \mathrm{q}$ in relation to survival does not seem to be CRC specific, but has also been reported for other 
epithelial tumors; i.e. bladder [31], head and neck [9], prostate [26] and breast cancer [33]. Deletion of $4 \mathrm{q}$ has also been associated with micro metastasis in lung cancer [46].

The critical region 4q22.1-35.1 encompasses $90.5 \mathrm{Mb}$ of chromosomal region with 265 coding genes. The size of the region thus makes it difficult to pin-point the candidate tumor suppressor genes or non-coding RNA. Integration of array CGH and gene expression array or extensive mutation analysis by next generation sequencing could possibly further narrow down the number of candidate driver genes.

In summary, loss of chromosome 4q22.1-35.1 may predict relapse of disease stage II colorectal cancer patients. Chromosome 4q may thus have important prognostic value for patient tailored therapy.

Acknowledgment This work was financially supported by the Dutch Cancer Society, grant KWF2007-3974

Open Access This article is distributed under the terms of the Creative Commons Attribution Noncommercial License which permits any noncommercial use, distribution, and reproduction in any medium, provided the original author(s) and source are credited.

\section{References}

1. F. Al-Mulla, S. AlFadhli, A.H. Al-Hakim, J.J. Going, M.S. Bitar, Metastatic recurrence of early-stage colorectal cancer is linked to loss of heterozygosity on chromosomes 4 and 14q. J. Clin. Pathol. 59, 624-630 (2006)

2. F. Al-Mulla, A.I. Behbehani, M.S. Bitar, G. Varadharaj, J.J. Going, Genetic profiling of stage I and II colorectal cancer may predict metastatic relapse. Mod. Pathol. 19, 648-658 (2006)

3. H.E. Alcock, T.J. Stephenson, J.A. Royds, D.W. Hammond, Analysis of colorectal tumor progression by microdissection and comparative genomic hybridization. Genes Chromosom. Cancer 37, 369-380 (2003)

4. A. Barrier, F. Roser, P.Y. Boelle, B. Franc, C. Tse, D. Brault, F. Lacaine, S. Houry, P. Callard, C. Penna, B. Debuire, A. Flahault, S. Dudoit, A. Lemoine, Prognosis of stage II colon cancer by nonneoplastic mucosa gene expression profiling. Oncogene 26, 2642-2648 (2007)

5. A.B. Benson III, D. Schrag, M.R. Somerfield, A.M. Cohen, A.T. Figueredo, P.J. Flynn, M.K. Krzyzanowska, J. Maroun, P. McAllister, C.E. Van, M. Brouwers, M. Charette, D.G. Haller, American society of clinical oncology recommendations on adjuvant chemotherapy for stage II colon cancer. J. Clin. Oncol. 22, 3408-3419 (2004)

6. T.E. Buffart, D. Israeli, M. Tijssen, S.J. Vosse, A. Mrsic, G.A. Meijer, B. Ylstra, Across array comparative genomic hybridization: a strategy to reduce reference channel hybridizations. Genes Chromosom. Cancer 47, 994-1004 (2008)

7. J. Camps, M. Grade, Q.T. Nguyen, P. Hormann, S. Becker, A.B. Hummon, V. Rodriguez, S. Chandrasekharappa, Y. Chen, M.J. Difilippantonio, H. Becker, B.M. Ghadimi, T. Ried, Chromosomal breakpoints in primary colon cancer cluster at sites of structural variants in the genome. Cancer Res. 68, 1284-1295 (2008)

8. B. Carvalho, C. Postma, S. Mongera, E. Hopmans, S. Diskin, M. A. van de Wiel, C.W. van, O. Thas, A. Matthai, M.A. Cuesta, J.S. Terhaar Sive Droste, M. Craanen, E. Schrock, B. Ylstra and G.A. Meijer, Multiple putative oncogenes at the chromosome 20q amplicon contribute to colorectal adenoma to carcinoma progression, Gut 58 (2009), 79-89

9. E. Cetin, B. Cengiz, E. Gunduz, M. Gunduz, H. Nagatsuka, L. Bekir-Beder, K. Fukushima, D. Pehlivan, N. MO, K. Nishizaki, K. Shimizu, N. Nagai, Deletion mapping of chromosome 4q22-35 and identification of four frequently deleted regions in head and neck cancers. Neoplasma 55, 299-304 (2008)

10. C. Compton, C.M. Fenoglio-Preiser, N. Pettigrew, L.P. Fielding, American joint committee on cancer prognostic factors consensus conference: colorectal working group. Cancer 88, 1739-1757 (2000)

11. P.M. De Angelis, T. Stokke, M. Beigi, O. Mjaland, O.P. Clausen, Prognostic significance of recurrent chromosomal aberrations detected by comparative genomic hybridization in sporadic colorectal cancer. Int. J. Colorectal Dis. 16, 38-45 (2001)

12. C.B. Diep, K. Kleivi, F.R. Ribeiro, M.R. Teixeira, O.C. Lindgjaerde, R.A. Lothe, The order of genetic events associated with colorectal cancer progression inferred from meta-analysis of copy number changes. Genes Chromosom. Cancer 45, 31-41 (2006)

13. A. Dupuy, R.M. Simon, Critical review of published microarray studies for cancer outcome and guidelines on statistical analysis and reporting. J. Natl Cancer Inst. 99, 147-157 (2007)

14. A. Figueredo, M.L. Charette, J. Maroun, M.C. Brouwers, L. Zuraw, Adjuvant therapy for stage II colon cancer: a systematic review from the cancer care Ontario program in evidence-based care's gastrointestinal cancer disease site group. J. Clin. Oncol. 22, 3395-3407 (2004)

15. K.S. Garman, C.R. Acharya, E. Edelman, M. Grade, J. Gaedcke, S. Sud, W. Barry, A.M. Diehl, D. Provenzale, G.S. Ginsburg, B. M. Ghadimi, T. Ried, J.R. Nevins, S. Mukherjee, D. Hsu, A. Potti, A genomic approach to colon cancer risk stratification yields biologic insights into therapeutic opportunities. Proc. Natl. Acad. Sci. U.S.A. 105, 19432-19437 (2008)

16. S. George, J. Primrose, R. Talbot, J. Smith, M. Mullee, D. Bailey, B.C. Du, H. Jordan, Will Rogers revisited: prospective observational study of survival of 3592 patients with colorectal cancer according to number of nodes examined by pathologists. Br. J. Cancer 95, 841-847 (2006)

17. S. Gill, C.L. Loprinzi, D.J. Sargent, S.D. Thome, S.R. Alberts, D. G. Haller, J. Benedetti, G. Francini, L.E. Shepherd, S.J. Francois, R. Labianca, W. Chen, S.S. Cha, M.P. Heldebrant, R.M. Goldberg, Pooled analysis of fluorouracil-based adjuvant therapy for stage II and III colon cancer: who benefits and by how much? J. Clin. Oncol. 22, 1797-1806 (2004)

18. M.Y. Kim, S.H. Yim, M.S. Kwon, T.M. Kim, S.H. Shin, H.M Kang, C. Lee, Y.J. Chung, Recurrent genomic alterations with impact on survival in colorectal cancer identified by genome-wide array comparative genomic hybridization. Gastroenterology 131, 1913-1924 (2006)

19. T. Knosel, K. Schluns, U. Stein, H. Schwabe, P.M. Schlag, M. Dietel, I. Petersen, Genetic imbalances with impact on survival in colorectal cancer patients. Histopathology 43, 323-331 (2003)

20. K. Kurashina, Y. Yamashita, T. Ueno, K. Koinuma, J. Ohashi, H. Horie, Y. Miyakura, T. Hamada, H. Haruta, H. Hatanaka, M. Soda, Y.L. Choi, S. Takada, Y. Yasuda, H. Nagai, H. Mano, Chromosome copy number analysis in screening for prognosisrelated genomic regions in colorectal carcinoma. Cancer Sci. 99, $1835-1840$ (2008)

21. G. Lanza, M. Matteuzzi, R. Gafa, E. Orvieto, I. Maestri, A. Santini, S.L. Del, Chromosome 18q allelic loss and prognosis in stage II and III colon cancer. Int. J. Cancer 79, 390-395 (1998)

22. G.J. Liefers, A.M. Cleton-Jansen, d. van, V, J. Hermans, J.H. van Krieken, C.J. Cornelisse and R.A. Tollenaar, Micrometastases and survival in stage II colorectal cancer, N.Engl.J.Med. 339 (1998), $223-228$ 
23. X.P. Liu, S. Kawauchi, A. Oga, T. Sato, K. Ikemoto, E. Ikeda, K. Sasaki, Chromosomal aberrations detected by comparative genomic hybridization predict outcome in patients with colorectal carcinoma. Oncol. Rep. 17, 261-267 (2007)

24. E. Mamounas, S. Wieand, N. Wolmark, H.D. Bear, J.N. Atkins, K. Song, J. Jones, H. Rockette, Comparative efficacy of adjuvant chemotherapy in patients with Dukes' B versus Dukes' C colon cancer: results from four National Surgical Adjuvant Breast and Bowel Project adjuvant studies (C-01, C-02, C-03, and C-04). J. Clin. Oncol. 17, 1349-1355 (1999)

25. E. Martinez-Lopez, A. Abad, A. Font, M. Monzo, I. Ojanguren, A. Pifarre, J.J. Sanchez, C. Martin, R. Rosell, Allelic loss on chromosome $18 \mathrm{q}$ as a prognostic marker in stage II colorectal cancer. Gastroenterology 114, 1180-1187 (1998)

26. S. Matsui, J. Laduca, M.R. Rossi, N.J. Nowak, J.K. Cowell, Molecular characterization of a consistent 4.5-megabase deletion at $4 \mathrm{q} 28$ in prostate cancer cells. Cancer Genet. Cytogenet. 159, 18-26 (2005)

27. G.A. Meijer, M.A. Hermsen, J.P. Baak, P.J. van Diest, S.G. Meuwissen, J.A. Belien, J.M. Hoovers, H. Joenje, P.J. Snijders, J. M. Walboomers, Progression from colorectal adenoma to carcinoma is associated with non-random chromosomal gains as detected by comparative genomic hybridisation. J. Clin. Pathol. 51, 901-909 (1998)

28. E. Morris, N.J. Maughan, D. Forman, P. Quirke, Who to treat with adjuvant therapy in Dukes B/Stage II colorectal cancer? - The need for high quality pathology. Gut 56, 1419-1425 (2007)

29. K. Nakao, K.R. Mehta, J. Fridlyand, D.H. Moore, A.N. Jain, A. Lafuente, J.W. Wiencke, J.P. Terdiman, F.M. Waldman, Highresolution analysis of DNA copy number alterations in colorectal cancer by array-based comparative genomic hybridization. Carcinogenesis 25, 1345-1357 (2004)

30. V.C. Petersen, K.J. Baxter, S.B. Love, N.A. Shepherd, Identification of objective pathological prognostic determinants and models of prognosis in Dukes' B colon cancer. Gut 51, 65-69 (2002)

31. T.J. Polascik, P. Cairns, W.Y. Chang, M.P. Schoenberg, D. Sidransky, Distinct regions of allelic loss on chromosome 4 in human primary bladder carcinoma. Cancer Res. 55, 5396-5399 (1995)

32. M. Sheffer, M.D. Bacolod, O. Zuk, S.F. Giardina, H. Pincas, F. Barany, P.B. Paty, W.L. Gerald, D.A. Notterman, E. Domany, Association of survival and disease progression with chromosomal instability: a genomic exploration of colorectal cancer. Proc. Natl. Acad. Sci. U.S.A. 106, 7131-7136 (2009)

33. N. Shivapurkar, S. Sood, I.I. Wistuba, A.K. Virmani, A. Maitra, S. Milchgrub, J.D. Minna, A.F. Gazdar, Multiple regions of chromosome 4 demonstrating allelic losses in breast carcinomas. Cancer Res. 59, 3576-3580 (1999)
34. L.H. Sobin, I.D. Fleming, TNM Classification of Malignant Tumors, fifth edition, Union internationale contre le cancer and the American joint committee on cancer. Cancer 80, 1803-1804 (1997)

35. K. Trautmann, J.P. Terdiman, A.J. French, R. Roydasgupta, N. Sein, S. Kakar, J. Fridlyand, A.M. Snijders, D.G. Albertson, S.N. Thibodeau, F.M. Waldman, Chromosomal instability in microsatellite-unstable and stable colon cancer. Clin. Cancer Res. 12, 6379-6385 (2006)

36. D. Tsafrir, M. Bacolod, Z. Selvanayagam, I. Tsafrir, J. Shia, Z. Zeng, H. Liu, C. Krier, R.F. Stengel, F. Barany, W.L. Gerald, P.B. Paty, E. Domany, D.A. Notterman, Relationship of gene expression and chromosomal abnormalities in colorectal cancer. Cancer Res. 66, 2129-2137 (2006)

37. M.A. van de Wiel, K.I. Kim, S.J. Vosse, W.N. van Wieringen, S. M. Wilting, B. Ylstra, CGHcall: calling aberrations for array CGH tumor profiles. Bioinformatics 23, 892-894 (2007)

38. M.A. van de Wiel, S.J. Smeets, R.H. Brakenhoff, B. Ylstra, CGHMultiArray: exact P-values for multi-array comparative genomic hybridization data. Bioinformatics 21, 3193-3194 (2005)

39. M.A. van de Wiel, W.N. van Wieringen, CGHregions: dimension reduction for array $\mathrm{CGH}$ data with minimal information loss. Cancer Inform. 3, 55-63 (2007)

40. L.N. van Steenbergen, V.E. Lemmens, M.J. Louwman, J.W. Straathof, J.W. Coebergh, Increasing incidence and decreasing mortality of colorectal cancer due to marked cohort effects in southern Netherlands. Eur. J. Cancer Prev. 18, 145-152 (2009)

41. A. Walther, R. Houlston, I. Tomlinson, Association between chromosomal instability and prognosis in colorectal cancer: a meta-analysis. Gut 57, 941-950 (2008)

42. M.M. Weiss, M.A. Hermsen, G.A. Meijer, N.C. van Grieken, J.P. Baak, E.J. Kuipers, P.J. van Diest, Comparative genomic hybridisation. Mol. Pathol. 52, 243-251 (1999)

43. J. Weitz, M. Koch, J. Debus, T. Hohler, P.R. Galle, M.W. Buchler, Colorectal cancer. Lancet 365, 153-165 (2005)

44. R. Winn, J. McClure, The NCCN clinical practice guidelines in oncology. J Natl Comprehensive Cancer Network 1, 5-13 (2003)

45. B.M. Wolpin, J.A. Meyerhardt, H.J. Mamon, R.J. Mayer, Adjuvant treatment of colorectal cancer. CA Cancer J. Clin. 57, $168-185$ (2007)

46. M. Wrage, S. Ruosaari, P.P. Eijk, J.T. Kaifi, J. Hollmen, E.F. Yekebas, J.R. Izbicki, R.H. Brakenhoff, T. Streichert, S. Riethdorf, M. Glatzel, B. Ylstra, K. Pantel, H. Wikman, Genomic profiles associated with early micrometastasis in lung cancer: relevance of 4q deletion. Clin. Cancer Res. 15, 1566-1574 (2009) 